\title{
927.
}

\section{ON CLIFFORD'S PAPER “ON SYZYGETIC RELATIONS AMONG THE POWERS OF LINEAR QUANTICS."}

[From the Proceedings of the London Mathematical Society, vol. xxIII. (1892), pp. $99-104$.

THE paper in question, originally printed, Proc. Lond. Math. Soc., t. III. (1869), pp. $9-12$, is reproduced No. xIV., pp. 119-122, of the Mathematical Papers (8vo. Lond. 1882), where it is immediately followed by the paper No. xv., "On Syzygetic Relations connecting the Powers of Linear Quantics," pp: 123-129. The author, after referring to theorems in M. Paul Serret's Géométrie de Direction (Paris, 1869), proceeds as follows:- "By the use of Professor Sylvester's method of contravariant differentiation, I have arrived at certain extensions of these theorems, which I now proceed to explain," and he then states his Theorem I.: In order that a system of $N$ points in a plane should all lie on a curve of the order $n$, it is sufficient that the $p$ th powers of their distances from an arbitrary line should satisfy a linear homogeneous relation, the number $N$ being given by the formula

$$
N=\frac{1}{2} \alpha n(n+3)+\frac{1}{2}(\beta+1)(\beta+2),
$$

where $\alpha$ is the quotient, and $\beta$ the remainder of the division of $p$ by $n$, so that $p=a n+\beta$, and $\beta<n$. And he then gives Theorem II., a like theorem as regards points in space; and, further, two Tables, $\mathrm{A}$ and $\mathrm{B}$, for the values of $N$ corresponding to given values of $n$ and $p$ in the two cases respectively.

Theorem I. is incorrect for the first value of $N$ in Table $\mathrm{A}$, viz. if $n=1, p=2$, then $N=5$; the theorem here is: In order that a system of five points in a plane may lie in a line, the sufficient condition is that the squares of their distances from an arbitrary line shall satisfy a linear homogeneous relation; or, what is the same thing, if the squares of the distances of the five points satisfy a linear homogeneous relation, then the five points will lie in a line. The right conclusion is that four of the five points will lie in a line. 
Before proceeding further, I slightly modify the form of the enunciation by defining (in the case of the plane figure) the power of a point to be its distance from an arbitrary line; or, what is the same thing, to be the nilfactum of the line-equation of the point; that is, for the point $\left(x_{1}, y_{1}, z_{1}\right)$, the power is

$$
\alpha x_{1}+\beta y_{1}+\gamma z_{1},
$$

where $\alpha, \beta, \gamma$ are arbitrary coefficients, or, if we please, line-coordinates. I say that $\alpha x_{1}+\beta y_{1}+\gamma z_{1}$ is the first power, $\left(\alpha x_{1}+\beta y_{1}+\gamma z_{1}\right)^{2}$ the second power, and so on. Clifford's Theorem I. thus is: If the $p$ th powers of the $N$ points satisfy a homogeneous linear relation, the $N$ points are on a curve of the $n$th order.

In the case $n=1, p=2$, we have five points whose second powers satisfy a bomogeneous linear relation; that is, if $\left(x_{1}, y_{1}, z_{1}\right), \ldots,\left(x_{5}, y_{5}, z_{5}\right)$ are the coordinates of the five points respectively, we have

$$
\lambda_{1}\left(\alpha x_{1}+\beta y_{1}+\gamma z_{1}\right)^{2}+\ldots+\lambda_{5}\left(\alpha x_{5}+\beta y_{5}+\gamma z_{5}\right)^{2}=0 .
$$

It is implicitly assumed that the points are distinct points, and we thus exclude such solutions as

$$
\lambda_{1}=\lambda_{2}=\lambda_{3}=0, \quad\left(x_{4}, y_{4}, z_{4}\right)=k\left(x_{5}, y_{5}, z_{5}\right), \quad k^{2} \lambda_{4}+\lambda_{5}=0 .
$$

Here $\alpha, \beta, \gamma$ are arbitrary, and the relation is equivalent to the six equations

$$
\begin{aligned}
\lambda_{1} x_{1}^{2}+\ldots+\lambda_{5} x_{5}^{2} & =0, \\
\lambda_{1} y_{1}^{2}+\ldots & =0, \\
\lambda_{1} z_{1}^{2}+\ldots & =0, \\
\lambda_{1} y_{1} z_{1}+\ldots & =0, \\
\lambda_{1} z_{1} x_{1}+\ldots & =0, \\
\lambda_{1} x_{1} y_{1}+\ldots & =0 ;
\end{aligned}
$$

and hence, eliminating the $\lambda$ 's, we have the relation

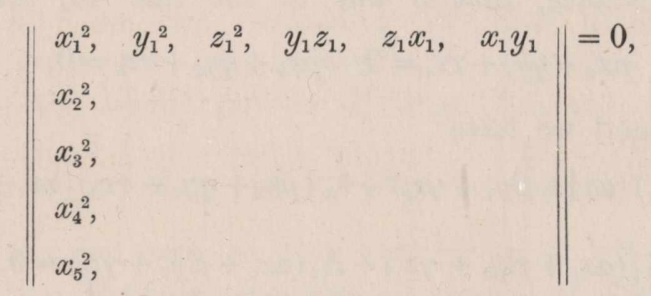

viz. this means that each of the determinants, obtained by selecting in any manner five out of the six columns, is $=0$. This is a twofold relation, and thus it cannot be equivalent to the threefold relation

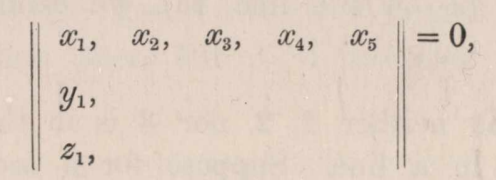

which expresses that the five points are in a line.

c. XIII. 
But we see further that the twofold relation expresses that the five points are such that we can, through them and an arbitrary sixth point $\left(x_{6}, y_{6}, z_{6}\right)$, draw a determinate conic; and this is the case only if four of the five points are in a line; viz. the conic is then the line-pair composed of this line and of the line joining the remaining two points. The right conclusion therefore is that, if the above linear relation is satisfied, then four of the five points lie in a line.

Clifford's proof is rather indicated than carried out, but, from the reference to contravariant differentiation, and from the second paper mentioned above, it must have been as follows:

Starting from the linear relation considered as an identity in $(\alpha, \beta, \gamma)$, and operating upon it with $p \partial_{\boldsymbol{a}}+q \partial_{\boldsymbol{\beta}}+r \partial_{\boldsymbol{\gamma}}, p, q, r$ denoting arbitrary coefficients, we obtain

$$
\lambda_{1}\left(p x_{1}+q y_{1}+r z_{1}\right)\left(\alpha x_{1}+\beta y_{1}+\gamma z_{1}\right)+\ldots+\lambda_{5}\left(p x_{5}+q y_{5}+r z_{5}\right)\left(\alpha x_{5}+\beta y_{5}+\gamma z_{5}\right)=0 ;
$$

hence, determining the ratios of $p, q, r$, say by the equations

$$
p x_{4}+q y_{4}+r z_{4}=0, \quad p x_{5}+q y_{5}+r z_{5}=0,
$$

and, instead of $\lambda_{1}\left(p x_{1}+q y_{1}+r z_{1}\right)$, \&c., writing $\Lambda_{1}$, \&c., we have

$$
\Lambda_{1}\left(\alpha x_{1}+\beta y_{1}+\gamma z_{1}\right)+\Lambda_{2}\left(\alpha x_{2}+\beta y_{2}+\gamma z_{2}\right)+\Lambda_{3}\left(\alpha x_{3}+\beta y_{3}+\gamma z_{3}\right)=0,
$$

a homogeneous linear relation between the first powers of the three points $\left(x_{1}, y_{1}, z_{1}\right)$, $\left(x_{2}, y_{2}, z_{2}\right),\left(x_{3}, y_{3}, z_{3}\right)$. We thus see that these points, say the points 1, 2, 3, are in a line; and, similarly, by different determinations of $p, q, r$, that the points $1,2,4$ are in a line; and that the points $1,2,5$ are in a line; that is, the points 3,4 and 5 are each of them in the line 12 joining the points 1 and 2; that is, the points $1,2,3,4,5$ are in a line.

But, if we examine the reasoning more closely, it appears that the first conclusion, 1, 2, 3 are in a line, depends on the assumption that neither 1,2 , nor 3 is in the line 45. Suppose, for instance, that 3 was in the line 45 , the equations

$$
p x_{4}+q y_{4}+r z_{4}=0, \quad p x_{5}+q y_{5}+r z_{5}=0,
$$

imply $p x_{3}+q y_{3}+r z_{3}=0$, and we have

$$
\lambda_{1}\left(p x_{1}+q y_{1}+r z_{1}\right)\left(\alpha x_{1}+\beta y_{1}+\gamma z_{1}\right)+\lambda_{2}\left(p x_{2}+q y_{2}+r z_{2}\right)\left(\alpha x_{2}+\beta y_{2}+\gamma z_{2}\right)=0,
$$

or say

satisfied by

$$
\Lambda_{1}\left(\alpha x_{1}+\beta y_{1}+\gamma z_{1}\right)+\Lambda_{2}\left(\alpha x_{2}+\beta y_{2}+\gamma z_{2}\right)=0,
$$

$$
\left(x_{1}, y_{1}, z_{1}\right)=k\left(x_{2}, y_{2}, z_{2}\right), k \Lambda_{1}+\Lambda_{2}=0,
$$

that is, by making the points 1 and 2 coincident. Thus, if 3 be on the line 45 (and similarly if 1 or if 2 be on the line 45), we cannot infer that $1,2,3$ are in a line.

I assume, therefore, that neither 1,2 , nor 3 is in the line 45 ; we here conclude, as above, that $1,2,3$ are in a line. Suppose for a moment that 5 is not on this line; 4 cannot be on each of the lines $15,25,35$, and I assume, in the first instance, 
that it is not on any one of these lines; thus the points 1, 2, 4 are no one of them on the line 35, and hence, by the like reasoning, 1, 2, 4 are in a line; that is, 4 is on the line 12 , or, the points $1,2,3,4$ are in a line. If, however, 4 is on one of the lines $15,25,35$, say it is on 35 ; then it is not on 25 , and no one of the points 1, 3, 4 is on 25; hence, by the like reasoning, 1, 2, 4 are in a line. In this case, however, 4 being, by supposition, on the line 35 , can only be the point 3 ; that is, 3 and 4 would be coincident, a case which need not be considered. The correct conclusion from the reasoning thus is, not that $1,2,3,4,5$ are in a line, but that some four of these five points, say 1, 2, 3, 4, are in a line.

It is easy to see that, if we have on a line four points, then there exists between the second powers of these points a linear homogeneous relation. For let the distances of the points from a fixed point of the line be $a, b, c, d$ respectively; and let the arbitrary line meet the line in a point $O$ at a distance $r$ from the fixed point. The distances of the four points from the point $O$ are thus equal to $r+a$, $r+b, r+c, r+d$ respectively; and hence, writing $k$ for the squared cosine of the inclination of the two lines, the second powers of the four points are $=k(r+a)^{2}$, $k(r+b)^{2}, \quad k(r+c)^{2}, \quad k(r+d)^{2}$ respectively; and we have thus, between the second powers $p_{1}, p_{2}, p_{3}, p_{4}$ of the four points, the homogeneous linear relation

$$
\left|\begin{array}{llll}
p_{1}, & 1, & a, & a^{2} \\
p_{2}, & 1, & b, & b^{2} \\
p_{3}, & 1, . & c, & c^{2} \\
p_{4}, & 1, & d, & d^{2}
\end{array}\right|=0
$$

Moreover we can, in an infinite number of ways, form a linear combination

$$
A_{1} p_{1}+A_{2} p_{2}+A_{3} p_{3}+A_{4} p_{4}
$$

of these second powers, which shall be independent of $r$, and have any given value whatever. We can therefore make this sum to be equal to the second power of any point 5 whatever (not on the line containing the four points) in relation to the arbitrary line; that is, given the four points in a line, and any other fifth point, we can establish between the second powers of these five points a linear homogeneous relation

$$
A_{1} p_{1}+A_{2} p_{2}+A_{3} p_{3}+A_{4} p_{4}+A_{5} p_{5}=0
$$

with non-evanescent values for each of the coefficients $A$. We thus see how, to the linear homogeneous relation between the second powers of the five points, there corresponds properly the non-symmetric relation: four of the five points are in a line.

The most simple cases are when $p=n$, viz. $p=n=2, N=6 ; p=n=3, N=10$, and generally $N=\frac{1}{2}(n+1)(n+2)$; and for these Clifford's theorem is easily verified. I have not examined the other cases, but it is probable that in each of them a correction is required. 